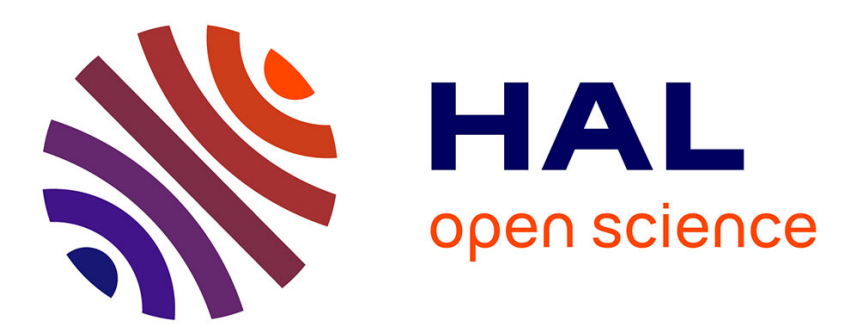

\title{
Experimental assessment of four ultrasound scattering models for characterizing concentrated tissue-mimicking phantoms
}

Emilie Franceschini, Régine Guillermin

\section{- To cite this version:}

Emilie Franceschini, Régine Guillermin. Experimental assessment of four ultrasound scattering models for characterizing concentrated tissue-mimicking phantoms. Journal of the Acoustical Society of America, 2012, 132 (6), pp.3735-3747. 10.1121/1.4765072 . hal-00770362

\section{HAL Id: hal-00770362 \\ https://hal.science/hal-00770362}

Submitted on 5 Jan 2013

HAL is a multi-disciplinary open access archive for the deposit and dissemination of scientific research documents, whether they are published or not. The documents may come from teaching and research institutions in France or abroad, or from public or private research centers.
L'archive ouverte pluridisciplinaire HAL, est destinée au dépôt et à la diffusion de documents scientifiques de niveau recherche, publiés ou non, émanant des établissements d'enseignement et de recherche français ou étrangers, des laboratoires publics ou privés. 
Experimental assessment of four ultrasound scattering models for characterizing concentrated tissue-mimicking phantoms

Emilie Franceschini and Régine Guillermin

Laboratoire de Mécanique et d'Acoustique LMA - CNRS UPR 7051, Aix-Marseille Université, Centrale Marseille, 13402 Marseille Cedex 20, France

(Dated: November 9, 2012) 


\begin{abstract}
Tissue-mimicking phantoms with high scatterer concentrations were examined using quantitative ultrasound techniques based on four scattering models: the Gaussian Model (GM), the Faran Model (FM), the Structure Factor Model (SFM) and the Particle Model (PM). Experiments were conducted using 10- and $17.5-\mathrm{MHz}$ focused transducers on tissue-mimicking phantoms with scatterer concentrations ranging from 1 to $25 \%$.

Theoretical BSCs were first compared with the experimentally measured BSCs in the forward problem framework. The measured BSC versus scatterer concentration relationship was predicted satisfactorily by the SFM and the PM. The FM and the PM overestimated the BSC magnitude at actual concentrations greater than $2.5 \%$ and $10 \%$, respectively. The SFM was the model that better matched the BSC magnitude at all the scatterer concentrations tested. Secondly, the four scattering models were compared in the inverse problem framework to estimate the scatterer size and concentration from the experimentally measured BSCs. The FM did not predict the concentration accurately at actual concentrations greater than $12.5 \%$. The SFM and PM need to be associated with another quantitative parameter to differentiate between low and high concentrations. In that case, the SFM predicted the concentration satisfactorily with relative errors below $38 \%$ at actual concentrations ranging from 10 to $25 \%$.
\end{abstract}

PACS numbers: 43.35.Bf 43.35.Yb 43.80 Vj 43.80.Cs

Keywords: ultrasound backscatter, ultrasound tissue characterization, structure factor, Percus-Yevick packing factor, Gaussian model 


\section{INTRODUCTION}

Quantitative ultrasound (QUS) techniques are based on the frequency-based analysis of the signals backscattered from biological tissues in order to determine the physical properties of the average tissue microstructures. These tissue characterization techniques aim to differentiate between diseased and healthy tissue and to detect cancer tumors. QUS techniques rely on theoretical scattering models in order to fit the spectrum of the echoes backscattered by biological tissues to an estimated spectrum using an appropriate model. The theoretical scattering model most frequently used for this purpose is the Gaussian Model (GM) developed by Lizzi ${ }^{1,2}$ which yields two tissue properties: the average scatterer size and the acoustic concentration (i.e., the product of the scatterer number density by the square of the relative impedance difference between the scatterers and the surrounding medium). This approach has been successfully used to characterize the eye ${ }^{3}$, the prostate ${ }^{4}$, the breast ${ }^{5-7}$, apoptotic cells ${ }^{8}$ and cancerous lymph nodes. ${ }^{9}$ Other theoretical scattering models such as the fluid-filled sphere model (FFSM) $)^{6,7}$ and the solid sphere model ${ }^{10}$ (which we refer to here as the Faran Model - FM) have also been used to predict average scatterer sizes by modeling the medium by an ensemble of fluid or solid spheres. In the aforementioned models (GM, FFSM and FM), the scatterers were assumed to be randomly distributed (i.e., to have a low scatterer concentration) and multiple scattering was neglected (in line with the Born approximation). Under these hypotheses, the power of the backscattered signals increases linearly with the scatterer concentration and depends on the size and acoustic properties of the tissue scattering structures. This linear relationship has been used to monitor the scatterer size and concentration.

However, the assumption that the scatterers are randomly distributed may not hold in tumors with densely packed cells. ${ }^{11}$ This means that a scattering theory involving a high cell concentration (i.e. accounting for a dense medium) should improve QUS techniques to determine the microstructural properties of tissues. Some theoretical efforts have been made in the field of ultrasonic blood characterization to take the high cell concentrations into 
account, ${ }^{12}$ since the concentration of red blood cells in blood (or hematocrit) ranges between 30 and $50 \%$. In the Rayleigh scattering regime (i.e., where the product of the wavenumber by the scatterer radius is $k a \ll 1)$, Twersky ${ }^{13}$ developed a Particle Model (PM) giving an expression for the backscattered intensity in terms of the single-particle backscattering cross section, the particle number density and the packing factor. The packing factor is a measure of orderliness in the spatial arrangement of particles. It depends on the cell concentration, but not on the frequency. The PM succeeded to explain the nonlinear relationship between the backscatter amplitude and the scatterer concentration in the case of non-aggregating red blood cells. ${ }^{14}$ Again in the field of blood characterization, the PM was later generalized to include any cell spatial distributions (i.e. aggregating red blood cell distributions) by introducing the frequency dependent structure factor, and called the Structure Factor model (SFM). ${ }^{15,16}$ The SFM sums the contributions from individual cells and models the cellular interaction by a statistical mechanics structure factor, which is defined as the Fourier transform of the spatial distribution of the cells. ${ }^{15,16}$ Note that the low frequency limit of the structure factor is by definition the packing factor used under Rayleigh conditions. ${ }^{13}$ Until quite recently, the PM and SFM were only used for blood characterization purposes, but Vlad et $a l^{11}$ have performed two-dimensional computer simulations based on the SFM in order to explain the backscatter behavior as a function of the particle size variance in the case of centrifuged cells undergoing cell death processes. These centrifuged cells mimicked the spatial distribution and packing of tumor cells. ${ }^{11}$

The aim of this study was to compare the backscatter coefficient (BSC) predictions given by four theoretical scattering models, namely the GM, FM, PM and SFM, with the BSCs measured experimentally on tissue-mimicking phantoms. These phantoms consisted of polyamide microspheres (mean radius $6 \mu \mathrm{m}$ ) suspended in water at various scatterer concentrations ranging from 1 to $25 \%$. The high scatterer concentrations were used to mimic densely packed cells in tumors. Ultrasonic backscatter measurements were performed at frequencies ranging from $6 \mathrm{MHz}$ to $22 \mathrm{MHz}$. The theoretical BSCs based on different scattering models were first compared with the BSCs measured experimentally in the forward problem 
framework, i.e. the theoretical BSCs were determined from known structural and acoustical properties of the polyamide microspheres. Secondly, comparisons were made between the four theoretical scattering models in the inverse problem framework to estimate the scatterer size and concentration from the experimentally measured BSCs. To our knowledge, the scatterer concentration has never been previously determined using the PM and SFM. Lastly, the validity of the PM and the SFM as means of determining scatterer concentrations is discussed.

\section{SCATTERING MODELS}

Four scattering models, the GM, FM, SFM and PM, are presented in this section. For all four scattering models, the formulations were written for monodisperse spheres and it was assumed that no multiple scattering occurred among the scatterers. When solving the inverse problem in the framework of each theory, the acoustical properties of spheres and the surrounding fluid were assumed to be known a priori and a minimization procedure was used to fit a curve to the measured BSC in order to estimate the size $a^{*}$ and concentration $\phi^{*}$ of the scatterers.

\section{A. The Gaussian model (GM)}

The BSC can be modeled using a spatial autocorrelation function describing the shape and distribution of the scatterers in the medium. The scattering sites are usually assumed to be randomly distributed and to have simple geometric shapes, which can be modeled in the form of gaussian scatterers representing continuous functions of changing impedance. In this framework, the BSC can be expressed as the product of the BSC in the Rayleigh limit and the backscatter form factor. ${ }^{17}$ The form factor describes the frequency dependence of the scattering, based on the size and shape of the scatterers. The Gaussian form factor has been used for many applications. ${ }^{3-9}$ It describes tissue structures as continuously varying distributions of acoustic impedance fluctuations about the mean value, and the effective 
radius is related to the impedance distribution of the scatterer.

The theoretical BSC used with the GM formulation is therefore written as follows: ${ }^{5,17}$

$$
B S C_{G M}(k)=\frac{k^{4} V_{s}^{2} n_{z}}{4 \pi^{2}} e^{-0.827 k^{2} a^{2}}
$$

where $k$ is the wave number, $V_{s}$ the sphere volume, $n_{z}$ the acoustic concentration and $a$ the mean effective radius of the scatterer.

The effective radius $a^{*}$ and the acoustic concentration $n_{z}^{*}$ were determined by comparing the logarithm of the experimental BSC values, denoted $B S C_{\text {exp }}$, with the logarithm of the theoretical $B S C_{G M}$ given by Eq. (1), as previously done by Oelze et al. ${ }^{6}$ Note that this fit was realized in the frequency bandwidth from 6 to $22 \mathrm{MHz}$.

\section{B. The Faran model (FM)}

The original theory developed by Faran ${ }^{18}$ provides an exact solution for the scattering of sound by a solid sphere in a surrounding fluid medium, and therefore includes shear waves in addition to compressional waves. The sphere is assumed to be insonified by a harmonic plane wave and to be located far from the point at which the scattered pressure field is observed. In the present study, the differential backscattering cross section at $180^{\circ}$ for a single scatterer $\sigma_{b}$ was computed for a sphere of radius $a$ using Faran's theory ${ }^{18}$ and then scaled by the number density to obtain the BSC from an ensemble of identical solid spheres as follows:

$$
B S C_{F M}(k)=\frac{\phi}{V_{s}} \sigma_{b}(k)
$$

where $\phi$ is the scatterer concentration. The ratio $\phi / V_{s}$, denoted $m$, corresponds to the number density of spheres.

The values of the unknown parameters were estimated by matching the experimentally measured $B S C_{\text {exp }}$ with the theoretical $B S C_{F M}$ given by Eq. (2). For this purpose, we searched for values of $(a, \phi) \in[0,50] \times[0.001,0.74]$, where $a$ was expressed in $\mu m$, minimizing 
the cost function:

$$
F_{F M}(a, \phi)=\sum_{i}\left\|B S C_{F M}\left(k_{i}\right)-B S C_{e x p}\left(k_{i}\right)\right\|^{2}
$$

Note that the maximum concentration was fixed to 0.74 , which corresponds to the closelypacked hexagonal arrangement. The cost function synthesizes all the wavenumbers $k_{i}(i=$ $1 \cdots N$ ) in the 6 to $22 \mathrm{MHz}$ frequency bandwidth. Figure 1(a) and (b) gives an example of a cost function surface $F_{F M}(a, \phi)$ obtained from the experiments performed with an actual microsphere radius of $6 \mu \mathrm{m}$ and an actual concentration of $2.5 \%$ presented below in section III. It can be seen from this figure that, at a given value of the radius, the cost functions have a single minimum. Figure 1(c) shows the gradient in the region of interest (ROI) around the values of the actual parameters.

Since there exists no analytical expression for $\sigma_{b}$ using Faran's theory, ${ }^{18}$ an exhaustive search was conducted on the value of $a$ in order to minimize the cost function $F_{F M}$ given by Eq. (3). In these studies, we started the exhaustive search in the 1 to $50 \mu m$ range with a $5 \mu \mathrm{m}$ step. The step was then decreased to $0.1 \mu \mathrm{m}$, while performing the search $10 \mu \mathrm{m}$ around the best value obtained so far. At each fixed value of the radius, the parameter $\phi$ was obtained using a trust-region-reflective algorithm with a minimization routine lsqnonlin in MATLAB (The MathWorks, Inc., Natick, MA).

\section{The Structure Factor Model (SFM) and the Particle Model (PM)}

The $\mathrm{SFM}^{15}$ is based on the assumption that at high scatterer concentrations, interference effects are mainly caused by correlations between the spatial positions of individual scatterers. The SFM has generally been applied to an ensemble of fluid spheres to model red blood cells in blood. ${ }^{15,16,19}$ Herein, the SFM was slightly adapted to the case of an ensemble of solid spheres which are homogeneously distributed in space. In comparison with the Faran model described in Eq. (2), the SFM considers the interference effects relatively easily by replacing the single-particle backscattering contribution $\sigma_{b}(k)$ by the product $\sigma_{b}(k) S(k)$, where $S(k)$ is the structure factor depending on the scatterer concentration and the pattern 
of the spatial arrangement of the scatterers. By considering an ensemble of identical solid spheres of radius $a$, the theoretical BSC for the SFM formulation is given by:

$$
B S C_{S F M}(k)=\frac{\phi}{V_{s}} \sigma_{b}(k) S(k)
$$

where the differential backscattering cross section $\sigma_{b}$ was calculated using the Faran's theory. The structure factor $S$ is related by definition to the three-dimensional (3D) Fourier transform of the total correlation function $(g(\mathbf{r})-1)$ as follows:

$$
S(k)=1+m \int(g(\mathbf{r})-1) e^{-2 j \mathbf{k r}} d \mathbf{r} .
$$

where $g(\mathbf{r})$ is the pair-correlation function, which is the probability of finding two particles separated by the distance $\mathbf{r}$. In this case, the structure factor can be interpreted as being a line of the 3D Fourier transform of the total correlation function $(g(\mathbf{r})-1)$, the line being in the direction of the incident wave (see appendix of Ref 20). The structure factor $S$ therefore depends only on the modulus $k$ of the wave vector $\mathbf{k}$. The structure factor cannot be calculated analytically for a complex spatial positioning of particles, as occurs in the case of aggregates of particles. However, for an ensemble of identical hard spheres homogeneously distributed, an analytical expression for the structure factor can be obtained, as established by Wertheim. ${ }^{21}$ The modified version of the analytical expression for the structure factor used here is described in detail in Appendix A.

In the low frequency limit, the structure factor tends towards a constant value $S(k) \rightarrow$ $S(0)=W$ called the packing factor. ${ }^{13}$ The most commonly used expression for the packing factor is based on the Percus-Yevick pair-correlation function for identical, hard and radially symmetric particles. The Perkus-Yevick packing factor $W_{P Y}$ is related with the scatterer concentration $\phi$ as follows ${ }^{13}$ :

$$
W_{P Y}=\frac{(1-\phi)^{4}}{(1+2 \phi)^{2}} .
$$

In comparison with the SFM described in Eq. (4), the theoretical BSC for the PM is thus obtained by replacing the structure factor $S$ by the Percus-Yevick packing factor $W_{P Y}$ as follows:

$$
B S C_{P M}(k)=\frac{\phi}{V_{s}} \frac{(1-\phi)^{4}}{(1+2 \phi)^{2}} \sigma_{b}(k) .
$$


Estimated values of $a^{*}$ and $\phi^{*}$ were determined by fitting the measured $B S C_{\exp }$ to the theoretical $B S C_{S F M}$ given by Eq. (4) in the case of the SFM (or with the theoretical $B S C_{P M}$ given by Eq. (7) in the case of the PM). For this purpose, we searched for values of $(a, \phi) \in[0,50] \times[0.001,0.74]$, where $a$ was expressed in $\mu m$, minimizing the cost function:

$$
\begin{aligned}
& F_{S F M}(a, \phi)=\sum_{i}\left\|B S C_{S F M}\left(k_{i}\right)-B S C_{e x p}\left(k_{i}\right)\right\|^{2}, \text { for the SFM } \\
& F_{P M}(a, \phi)=\sum_{i}\left\|B S C_{P M}\left(k_{i}\right)-B S C_{e x p}\left(k_{i}\right)\right\|^{2}, \text { for the PM. }
\end{aligned}
$$

The cost function had several local minima, as observed by plotting the cost function surfaces. Figure 2(a) and (b) gives an example of a cost function surface $F_{S F M}(a, \phi)$ for the SFM obtained from the experiments performed with an actual microsphere radius of $6 \mu m$ and an actual concentration of $20 \%$ described below in section III. Similar behavior was observed in the case of the PM. One can observe that, at a given value of the radius, the cost functions show either one or two minima. In the case of two minima, one minimum corresponds to a low scatterer concentration estimate and the other one to a high concentration estimate. This point will be discussed below in section V.C. Figure 2(c) shows the gradient in the ROI around the values of the actual parameters.

The same procedure was used to estimate $a^{*}$ and $\phi^{*}$ with the SFM and the PM. Since no analytical expression for $\sigma_{b}$ is available in the framework of Faran's theory, ${ }^{18}$ as mentioned above, an exhaustive search was conducted on the values of $a$ in the 1 to $50 \mu m$ range in order to minimize the cost function $F_{S F M}$ (or $F_{P M}$ ) given by Eq. (8). Since the cost functions can display two minima, we searched for two concentration estimates $\phi_{1}$ and $\phi_{2}$ at each given value of $a$, using a trust-region-reflective algorithm with the MATLAB nonlinear data-fitting lsqnonlin (The MathWorks, Inc., Natick, MA). The concentration value obtained $\phi_{1}$ (or $\phi_{2}$, respectively) corresponds to the minimum of the cost function obtained with an initial concentration of $\phi^{\text {init }}=0.001$ (or $\phi^{\text {init }}=0.74$ ) at the beginning of the minimization routine. By varying the value of $a$, two possible solutions $\left(a_{1}^{*}, \phi_{1}^{*}\right)$ and $\left(a_{2}^{*}, \phi_{2}^{*}\right)$ were obtained, 
corresponding to a low initial value and a high initial value of the concentration at the beginning of the minimization routine. The solution $\left(a^{*}, \phi^{*}\right)$ corresponds to the minimum value between $F_{S F M}\left(a_{1}^{*}, \phi_{1}^{*}\right)$ and $F_{S F M}\left(a_{2}^{*}, \phi_{2}^{*}\right)$ with the SFM (or equivalently $F_{P M}\left(a_{1}^{*}, \phi_{1}^{*}\right)$ and $F_{P M}\left(a_{2}^{*}, \phi_{2}^{*}\right)$ with the PM). Note that in some cases, the values of $F_{S F M}\left(a_{1}^{*}, \phi_{1}^{*}\right)$ and $F_{S F M}\left(a_{2}^{*}, \phi_{2}^{*}\right)$ (or equivalently the values of $F_{P M}\left(a_{1}^{*}, \phi_{1}^{*}\right)$ and $F_{P M}\left(a_{2}^{*}, \phi_{2}^{*}\right)$ for the PM) are equal.

\section{METHODS}

\section{A. Tissue-mimicking phantoms}

The tissue-mimicking phantoms consisted of polyamide microspheres with a radius of $6 \pm 2 \mu \mathrm{m}$ (orgasol 2001 EXD NAT1, Arkema, France) gently stirred in water. The size distribution of the microspheres measured using optical microscopy is presented in Fig. 3. The tissue-mimicking phantoms usually consisted of microspheres in agar-agar phantom. In the present study, the microspheres were suspended in water because agar-agar phantoms consisting of polyamide microspheres are difficult to degas at high scatterer concentration $(>15 \%)$. The phantoms had identical scatterer sizes but different scatterer concentrations ranging from 1 to $25 \%$. The acoustic parameters (the sound speed $c$, density $\rho$, impedance $z$ and Poisson's ratio) of the polyamide microspheres are given in Table I. The tissue-mimicking phantoms were designed to mimick the structural and acoustical properties of densely packed cells in tumors. The properties of the cell components are not well known and vary widely in the literature. Table I gives an example of cell properties used by Baddour and Kolios ${ }^{22}$ and Doyle et al. ${ }^{23}$ Note that the impedance and Poisson's ratio of polyamide microspheres are similar to those of human acute myeloid leukemia cell nuclei.

\section{B. Experimental setup}

Two broadband focused transducers with center frequencies of $10 \mathrm{MHz}$ and $17.5 \mathrm{MHz}$ (and focuses of $14.2 \mathrm{~mm}$ and $13.8 \mathrm{~mm}$, respectively) were used in these experiments. The 
pulse-echo acquisition system was composed of an Olympus model 5072 PR pulser-receiver and a Gagescope model 8500CS acquisition board. The transducer was placed in an agaragar gel, i.e. a solidified mixture of distilled water and 2\% (w/w) agar powder (A9799, Sigma Aldrich, France) so that the distance between the transducer and the suspension was equal to $13.2 \mathrm{~mm}$ in the $10 \mathrm{MHz}$ experiment (and $12.8 \mathrm{~mm}$ in the $17.5 \mathrm{MHz}$ experiment). The transducer focus was therefore positioned below the agar-agar/suspension interface at a distance of $1 \mathrm{~mm}$. The suspension was stirred in a beaker with a magnetic agitator to prevent sedimentation. Sixty RF lines were acquired and stored. Echoes were selected in the focal zone with a rectangular window $d=0.13 \mathrm{~cm}$ in length. The power spectra of the backscattered RF echoes were then averaged to provide $\overline{P_{\text {meas }}}$. This procedure was repeated twice with the two transducers at each scatterer concentration.

\section{Attenuation measurements}

The attenuation coefficients of the tissue-mimicking phantoms were determined using a standard substitution method. The $10 \mathrm{MHz}$ transducer was used in the reflection mode with a reflector on the opposite side, as shown in Fig. 4. At each phantom concentration, acquisitions of $100 \mathrm{rf}$ lines were performed both with the suspension and with water. The water acquisition was used for normalization. At each acquisition, the phantom attenuation was calculated using log spectral difference technique. ${ }^{24}$ These values were then averaged to obtain $\alpha_{p h}$ and are summarized in Fig. 5.

\section{BSC estimation}

The measured BSC values reported in this study were computed using the normalization technique for focused transducers described by Wang and Shung. ${ }^{25}$ This normalization technique consists in using a reference scattering medium instead of a perfect flat reflector, on condition that the BSC of the reference scattering medium is known or can be determined. ${ }^{25}$ The reference scattering medium allows to compensate the measured backscattered power 
spectrum $\overline{P_{\text {meas }}}$ for the electromechanical system response and the depth-dependent diffraction and focusing effects caused by the ultrasound beam. In the case of blood ultrasound characterization, the reference scattering medium is generally a low $6 \%$ hematocrit red blood cell suspension. ${ }^{25,26}$ Since the radius of one red blood cell sphere-shaped model is around 2.75 $\mu \mathrm{m}$, the corresponding theoretical BSC is given by the classical version of the PM written for an ensemble of identical Rayleigh scatterers (see Eq. (7) in Ref. 26). In the present study, the reference scattering medium used was a suspension of polyamide microspheres with a radius of $2.5 \mu \mathrm{m}$ (orgasol 2001 UD NAT1, Arkema, France) present at a low concentration of $0.5 \%$ and gently stirred in water. The sample is easy to prepare and to handle, and the scattering process occurring in an ensemble of identical solid microspheres at a very low concentration (dilute medium) has been well documented using the FM. ${ }^{18,27}$ Note that a small scatterer size of $2.5 \mu \mathrm{m}$ was chosen so that the reference scattering medium would be in the Rayleigh scattering regime $(k a \ll 1)$ and to avoid the resonant peaks that occur with higher scatterer sizes. Echoes from the reference scattering medium were windowed as with the tissue-mimicking phantoms at the same depth, and sixty echoes were also averaged to obtain $\overline{P_{\text {ref }}}$. The measured BSC was thus computed as follows ${ }^{25,26}$

$$
B S C_{\text {exp }}(k)=B S C_{\text {ref }}(k) \frac{\overline{P_{\text {meas }}(k)}}{\overline{P_{\text {ref }}(k)}} e^{\frac{4}{8.68}\left(\alpha_{p h} d\right) k \frac{c}{2 \pi}}=B S C_{r e f}(k) \frac{\overline{P_{\text {meas }}(k)}}{\overline{P_{\text {ref }}(k)}} e^{\frac{4}{8.68}\left(\alpha_{p h} d\right) f}
$$

where $f$ is the frequency in $\mathrm{MHz}$ and the theoretical BSC of the reference sample $B S C_{r e f}$ is given by Eq. (2) using the FM. Note that the last term in Eq. (9) compensates the measured $B S C_{\text {exp }}$ for the predetermined phantom attenuation values $\alpha_{p h}$ (in $\mathrm{dB} / \mathrm{cm} / \mathrm{MHz}$ ). The coefficient 8.68 expresses unit conversion from dB to Neper: $\alpha_{p h}[\mathrm{Neper} / \mathrm{cm} / \mathrm{MHz}]=$ $\alpha_{p h}[\mathrm{~dB} / \mathrm{cm} / \mathrm{MHz}] / 8.68$.

\section{RESULTS}

\section{A. Forward problem: comparison between theoretical and experimental results}

Figures 6 and 7 give the measured $B S C_{\text {exp }}$ versus the frequency at scatterer concentrations of $1,5,10,15$ and $25 \%$. The solid lines give the $B S C_{\text {exp }}$ values based on measurements 
performed with the 10-MHz center frequency transducer in the 6-15 MHz frequency bandwidth and with the $17.5-\mathrm{MHz}$ center frequency transducer in the 10-22 $\mathrm{MHz}$ frequency bandwidth. At all the concentrations tested, the $B S C_{\exp }$ measured with the two transducers in the 10-15 MHz frequency bandwidth were similar. This means that the results were not influenced by the system's transfer function. Fig. 6(a) and 7(a) also gives the theoretical BSC predictions based on the FM, and Fig. 6(b) and 7(b) gives the theoretical BSC predictions based on the SFM and PM. At all the scatterer concentrations tested, the best agreement between the experimental $B S C_{\text {exp }}$ and theoretical BSCs was obtained with the SFM in the 6-15 MHz frequency bandwidth. Whereas the results obtained with the FM were only satisfactory at the $1 \%$ scatterer concentration, and those obtained with the PM were satisfactory at concentrations of 1, 5, 10 and 15\%. Note that the results obtained with the GM were not presented in the forward problem study, since the GM does not include shear wave in the modeling. The GM therefore gave less satisfactory results that the FM (data not shown), even at the lowest concentration of $1 \%$.

To enhance the lecture of these results, Figure 8 shows the measured $B S C_{\text {exp }}$ magnitude averaged in the frequency bandwidth from 6 to $15 \mathrm{MHz}$ and in the frequency bandwidth from 15 to $22 \mathrm{MHz}$ as a function of the scatterer concentration. The standard deviation plotted in Fig. 8 was computed as described below. The power spectra of ten backscattered $\mathrm{RF}$ echoes were averaged to obtain $\overline{P_{\text {meas }}^{\prime}}$. Six power spectra $\overline{P_{\text {meas }}^{\prime}}$ were thus obtained from all sixty RF echoes acquired, and the six corresponding $B S C_{\text {exp }}^{\prime}$ were calculated using Eq. (9). The standard deviation of the $B S C_{e x p}^{\prime}$ averaged over the frequency bandwidth was then computed. Note that the $B S C_{\text {exp }}$ magnitude changed with the concentration: the $B S C_{\text {exp }}$ magnitude increased with increasing concentration between 1 and 10\%, then decreased with increasing concentration between 10 and 25\%. Also plotted in Fig. 8 are the theoretical BSC values predicted with the FM, SFM and PM. Good agreement was obtained at a low scatterer concentration of $1 \%$ and $2.5 \%$ for all models. The FM and the PM overestimated the BSC amplitude at $\phi>2.5 \%$ and for $\phi>10 \%$, respectively. The SFM was the model showing the best agreement with the experimental data, especially in the 
6-15 MHz frequency bandwidth. The SFM slightly overestimates the BSC magnitude at $\phi>10 \%$ in the $15-22 \mathrm{MHz}$ frequency bandwidth.

A quantitative ultrasonic parameter that has often been used for tissue characterization is the spectral slope (SS). The SS is the linear slope of the BSC as a function of the frequency on a log-log scale. The changes in the SS in the 6-22 MHz frequency bandwidth with the actual concentration are shown in Fig. 9. The experimental data did not show the occurrence of any significant changes in the frequency dependence of the $B S C_{\text {exp }}$ at the various scatterer concentrations tested, since the SS varies randomly between 3.4 and 3.7. The SSs obtained with the three scatterings models were all in the same range of values. The SSs of the FM and the PM were equal to a constant value of 3.44 at all the concentrations tested, whereas the SS of the SFM slightly increased from 3.46 to 3.75 with increasing concentrations.

\section{B. Inverse problem: radius and concentration estimates}

In what follows, the relative errors for parameters $a^{*}$ and $\phi^{*}$ correspond to:

$$
\epsilon_{a^{*}}=\frac{a^{*}-a}{a} \quad \epsilon_{\phi^{*}}=\frac{\phi^{*}-\phi}{\phi}
$$

\section{Radius and concentration estimates using the GM and FM}

The estimated values $a^{*}$ and $\phi^{*}$ using the GM and the FM and the corresponding relative errors are given in Fig. 10. The results obtained with the GM are presented in the inverse problem framework because that model has been widely used in previous studies. $^{3-9}$ Since the impedance difference $\gamma_{z}$ between the microspheres and the water is

known a priori, the estimated concentration $\phi^{*}=\frac{n_{z}^{*}(4 / 3) \pi a^{* 3}}{\gamma_{z}^{2}}$ with the GM is given here instead of the estimated acoustic concentration $n_{z}^{*}$ usually given in previous studies. ${ }^{5,6}$ The GM overestimated the microsphere radii: the average effective radii estimated at all the concentrations was found equal to $10.43 \pm 1.65 \mu \mathrm{m}$, corresponding to a relative error of $74 \pm 27 \%$. The GM therefore underestimated the scatterer concentrations, giving relative errors between $88 \%$ and $100 \%$ (average relative errors $90 \pm 9 \%$ ) at all the actual 
concentrations tested. The results obtained with the GM were somewhat anticipated, since this model is well adapted for fluid spheres in a dilute medium and is therefore not very suitable for modeling solid spheres in a dense medium.

The radii estimated using the FM were quantitatively satisfactory at all the actual concentrations tested, since relative absolute errors of less than $22 \%$ were obtained, except for the actual concentration of $5 \%$, at which a relative error of approximately $38 \%$ occurred (see Fig. 10). At concentrations of 1, 2.5, 7.5, 10 and 12.5\%, the estimated concentrations given by the FM were qualitatively consistent with a significant correlation with $r^{2}$ of 0.96 between the true and estimated concentrations (data not shown). The estimated concentrations were quantitatively satisfactory for the actual concentrations 7.5, 10 and $12.5 \%$ with relative errors inferior to $23 \%$. Note that at the actual concentration of $5 \%$, underestimating the radius resulted in overestimating the concentration. The FM concentrations were underestimated at the highest actual concentrations, i.e., those greater than $12.5 \%$, as it was expected.

\section{Radius and concentration estimates using the SFM and PM}

The results obtained using the SFM are shown in Fig. 11. The triangular symbols (and the diamond-shaped symbols) represent the possible solutions $\left(a_{1}^{*}, \phi_{1}^{*}\right)$ (and the possible solutions $\left(a_{2}^{*}, \phi_{2}^{*}\right)$, respectively) given by the SFM with an initial concentration of $\phi^{\text {init }}=0.001$ (and $\phi^{\text {init }}=0.74$, respectively) at the beginning of the minimization routine. The SFM gave quantitatively satisfactory radius estimates $a_{1}^{*}$ and $a_{2}^{*}$ at the concentrations studied with relative absolute errors of less than $20 \%$. The estimated concentrations $\phi_{1}^{*}$ obtained with a low initial concentration $\phi^{\text {init }}=0.001$ were quantitatively satisfactory, giving relative absolute errors of less than $31 \%$ at actual concentrations ranging from 5 to $15 \%$, and were qualitatively consistent at the two lowest concentrations of 1 and $2.5 \%$, giving relative absolute errors of $77 \%$ and $58 \%$, respectively (see the triangular symbols in Fig. 11(a2) and 11(b2)). The estimated concentrations $\phi_{2}^{*}$ obtained with a high initial 
concentration $\phi^{\text {init }}=0.74$ were quantitatively satisfactory giving relative absolute errors of less than $38 \%$ at actual concentrations ranging from 10 to $25 \%$ (see the diamond-shaped symbols in Fig. 11(a2) and 11(b2)). The circular symbols in Fig. 11 give the final solution $\left(a^{*}, \phi^{*}\right)$ corresponding to the global minimum of the cost function studied. Note that the global minimum $\left(a^{*}, \phi^{*}\right)$ did not always correspond to the actual parameters, whereas the value of one of the two local minima $\left(a_{1}^{*}, \phi_{1}^{*}\right)$ or $\left(a_{2}^{*}, \phi_{2}^{*}\right)$ is closed from the actual parameters.

Figure 12 presents the results obtained using the PM. These results were fairly similar to those obtained using the SFM. Note that the PM yielded less satisfactory concentrations $\phi_{2}^{*}$ with relative errors of up to $105 \%$ at the highest concentrations, i.e., those greater than 15\% (see the diamond-shaped symbols in Figs. 12(a2) and 12(b2)). But the estimated concentrations $\phi_{1}^{*}$ were more accurate at the low concentrations 1 and $2.5 \%$, giving relative absolute errors of less than $32 \%$.

\section{DISCUSSION}

\section{A. Forward problem: discussion}

BSCs in the 6 to $22 \mathrm{MHz}$ frequency range were measured using tissue-mimicking phantoms at scatterer concentrations ranging from 1 to $25 \%$. The experimental data thus obtained were compared with the predictions of three ultrasound scattering models, namely the FM, PM and SFM, in the framework of a forward problem study. This study is in line with that performed by Chen and Zagzebski ${ }^{31}$ using Sephadex spheres immersed in gel. In the latter study, qualitative comparison were made between the experimental and theoretical BSC behavior as a function of the scatterer concentration (see Figures 1 and 8 in Ref. 30) using a continuum scattering model. In the present study, the relationship between the BSC magnitude and scatterer concentration was studied quantitatively. The observed BSC magnitude versus concentration relationship was satisfactorily predicted by the SFM and the PM, whereas the FM predicted that the BSC increases with increasing concentrations. 
With the FM, the assumption that the scatterers were randomly distributed failed due to the spatial correlation among the scatterers at high scatterer concentrations. The FM therefore failed to predict the BSC magnitude at scatterer concentrations above $2.5 \%$. The $B S C_{P M}$ curves were in good agreement with the $B S C_{\text {exp }}$ curves up to concentrations of $15 \%$ (see Fig. 8). But the best agreement between the theoretical and experimental BSC curves in scattering magnitude was obtained with the SFM, even at the highest concentration of more than $15 \%$, especially in the 6-15 MHz frequency bandwidth (see Fig. 8(a)). To conclude, the SFM was found to be more suitable than the PM and the FM for modeling high scatterer concentrations.

Concerning the frequency dependence of the BSC, the relationship between the SS and the scatterer concentration was also studied. Since the SS values obtained on the basis of the experimental data varied randomly around 3.55 at the various concentrations tested, there is no clear variation of the SS with the concentration. The experimental SSs did not match the theoretical SSs given by the FM, SFM and PM. Contrary to what occurred with the experimental values, the theoretical SSs obtained with the FM and PM are constant as a function of the concentration, since the BSC frequency dependence with these models is determined only by the frequency dependence of $\sigma_{b}$ (see equations (2) and (7)). Note that $\sigma_{b}$ depends on the scatterer size and not on the concentration. With the SFM, the SS was predicted to increase with the concentration because of the structure factor. Indeed, according to Eq. (4), the $B S C_{S F M}$ frequency dependence is determined by the frequency dependences of $\sigma_{b}$ and $S$. Note that $S$ depends on the scatterers' radius and their concentration. However, the experimental and theoretical SSs values obtained ranged between 3.4 and 3.75 with all three models.

\section{B. On the use of polyamide microspheres to make cell-mimicking phantoms}

In the present study, experiments were performed at the cell scale using solid polyamide microspheres with a radius of $6 \mu \mathrm{m}$, having similar acoustic parameters to those of isolated 
nuclei (see Table I). However, the suitability of models for describing scattering by cells has given rise to some debate. Cells have also been modeled as fluid media. Two other approaches have been used so far to modeling a cell: using a single $\mathrm{FFSM}^{28}$ or modeling two concentric fluid spheres ${ }^{29}$ with impedances of around 1.6 MRayl. However, weakly scattering fluid phantoms (i.e., with impedances of around 1.6 MRay) consisting of small microspheres are difficult to manufacture. The smallest weakly scattering fluid microspheres found in the literature were around $90-212 \mu \mathrm{m}$ in size. ${ }^{30}$ This means that only large scale experiments (using low frequencies) can be performed with these weakly scattering fluid microspheres.

Besides the choice of an elastic or fluid medium for modeling cells, another disadvantage of these tissue-mimicking phantoms is the large impedance contrast involved: that between polyamide microspheres and water is around $58 \%$, versus $30 \%$ between cell nuclei and cytoplasm/extracellular matrix (taking $c=1570 \mathrm{~m} / \mathrm{s}, \rho=1.06$ and $z=1.66$ MRayl for the cytoplasm and the extracellular matrix and $z=2.15$ MRayl for nuclei as given in Table I). Although the wave propagation distance was quite short in our experiments $(d=1.3 \mathrm{~mm})$, multiple scattering can occur at high scatterer concentrations, which may explain the discrepancies observed between the measured $B S C_{\text {exp }}$ and theoretical $B S C_{S F M}$ in the $15-22$ $\mathrm{MHz}$ range (see Fig. 8(b)).

\section{Inverse problem}

In the forward problem study, it was established that the SFM was the model giving the best match with the experiments at all the concentrations tested. However, the best model for use with inverse problems should not only give a good fit with the data but also should be associated with a highly variable cost function in the ROI. It is worth noting that the cost function of the SFM has a higher gradient than the FM in the ROI, and that the area in which the gradient is small is larger in the case of the FM (see Fig. 1(c) and 2(c)). In addition, at a given value of the radius, the FM cost function minima all have similar values, whereas the SFM cost function minima are extremely variable (see Fig. 1(b) 
and 2(b)). The behavior of the cost functions shows that the SFM is more sensitive than the FM to the unknown parameters, which could be an important point when dealing with the noisy data obtained in the case of real tissue.

The accuracy of the FM to estimate the scatterer size and concentration was first examined. As can be seen from Fig. 10, the FM does not give accurate scatterer concentrations at $\phi>12.5 \%$. However, good correlations between the scatterer concentration predicted by the FM and the actual concentration were found at actual concentrations ranging between 1 and $12.5 \%$ (apart from 5\%). We did not actually expect to obtain such good results up to $12.5 \%$, since the FM overestimates the BSC amplitude for $\phi>2.5 \%$ as shown in the forward problem study. It is worth noting that the concentration estimates obtained at actual concentrations ranging between $7.5 \%$ and $12.5 \%$ were associated with a slight underestimation of the scatterer radius, which may explain why such accurate results were obtained at these actual concentrations.

The accuracy of the SFM and the PM to estimate the scatterer size and concentration was then examined in the case of high scatterer concentration, since the PM and the SFM are more suitable than the FM for modeling dense media. To our knowledge, neither the PM nor the SFM have been tested so far in blood characterization studies as means of predicting the scatterer concentration because the hematocrit is assumed to be known $a$ priori. ${ }^{26}$ However, our study showed that the estimation of the scatterer concentration is not straightforward using the PM or the SFM. The following discussion intends to explain why the concentration estimates cannot be obtained directly using standard minimization procedures in the 6-22 MHz frequency bandwidth studied here.

As can be seen in Fig. 8(a), when the value of the radius is fixed, the BSC magnitude can be the same at both small and large scatterer concentrations (for example, at the concentrations $5 \%$ and $20 \%$ in the $6-15 \mathrm{MHz}$ frequency bandwidth), apart from the maximum BSC magnitude, at which the concentration is unique (herein, 10\%). The $B S C_{S F M}$ 
magnitudes computed with the SFM versus the concentration are plotted in Fig. 13(a) at several radius sizes (ranging from 5 to $7 \mu \mathrm{m}$ ). It can be observed here that several pairs of parameters $(a, \phi)$ can have the same BSC magnitude. This explains why the cost functions of the SFM and the PM had several local minima, as shown in Fig. 2. In Figures 11 and 12 , one can notice that the global minimum $\left(a^{*}, \phi^{*}\right)$ did not always correspond to the actual parameters. However, one of the two local minima $\left(a_{1}^{*}, \phi_{1}^{*}\right)$ or $\left(a_{2}^{*}, \phi_{2}^{*}\right)$ corresponded to the actual parameters. To better understand what happened in these special cases, Fig. 13(b) gives the experimental $B S C_{\text {exp }}$ at a concentration of $20 \%$ and two fitted curves $B S C_{S F M}$ corresponding to the two local minima. Although the curves fitted with the SFM were similar, the concentration estimates were very different : $\left(a_{1}^{*}=6.5 \mu m, \phi_{1}^{*}=5.69 \%\right)$ and $\left(a_{2}^{*}=6.9\right.$ $\mu m, \phi_{2}^{*}=22.50 \%$ ). In this example, the global minimum was obtained with the smallest concentration (i.e. $\phi^{*}=\phi_{1}^{*}=5.69 \%$ ), whereas the actual concentration was equal to $20 \%$. This means that the information used to solve the inverse problem did not suffice to be able to determine the concentration. The discussion next considers some possible solutions to this problem.

One solution might be to increase the information available by using higher frequencies. Figure 13(c) shows the theoretical $B S C_{S F M}$ in the 6 to $40 \mathrm{MHz}$ frequency bandwidth at concentrations of $5 \%$ and $20 \%$ with radii of $5.5 \mu \mathrm{m}$ and $6 \mu \mathrm{m}$. With the same fixed radius, the $B S C_{S F M}$ versus frequency curves obtained at both scatterer concentrations share a similar pattern, since the peaks and dips occur at the same frequencies. In addition, at the same fixed radius, the $B S C_{S F M}$ amplitudes are practically identical at low frequencies $(<20 \mathrm{MHz})$, whereas the $B S C_{S F M}$ amplitude is larger at high frequencies $(>20 \mathrm{MHz})$ for the highest concentration of $20 \%$. The use of higher frequencies may thus (1) improve the radius estimates, since the BSC peaks and dips could be captured in the $25-40 \mathrm{MHz}$ frequency bandwidth, and (2) increase the ability to differentiate between low and high concentrations, since the BSC amplitude differs between low and high concentrations at higher frequencies. The use of high frequencies with QUS techniques could be envisaged when working with subcutaneous or excised tissues, as in the study performed by Mamou et $a l^{9}$ on cancer 
patients' freshly-dissected lymph nodes. However, if one keeps a low frequency bandwidth (6 - $22 \mathrm{MHz}$ as performed in our in vitro study or even less) with a view to develop in vivo SFM applications, the microstructure (i.e. the scatterer size and concentration) estimations based on the SFM might be associated with another quantitative parameter, such as the attenuation. In the tissue-mimicking phantom study presented here, the attenuation was found to increase with the concentration, as shown in Fig. 5 and this information could be used to choose a solution between the two local minima. This means that if the attenuation is high, one can expect to have high scatterer concentrations. Moreover, in the case of some tissues such as liver or most cancer tissues, the cells are known to be densely packed. Structural parameter estimations based on the SFM could be possibly limited to a high initial concentration value of $\phi^{i n i t}=0.74$ at the beginning of the minimization routine. It is worth emphasizing that, by combining the structural estimates with the attenuation or by assuming a priori that the medium is dense, the SFM yielded satisfactory concentration estimates with relative errors of less than $38 \%$ at actual concentrations ranging from 10 to $25 \%$.

\section{CONCLUSION}

Four scattering models were examined for characterizing biological tissues with high scatterer concentrations. The GM has been previously used in various tissue studies; $;^{3-9}$ whereas the FM has been mainly used for tissue-mimicking phantoms composed of solid particles. ${ }^{10,31}$ Both of these models assume randomly distributed scatterers such that the theoretical BSC magnitude increases with scatterer concentration. These models are therefore most suitable for dealing with dilute media. The SFM and PM have been developed for modeling dense blood medium ${ }^{13-16}$ in order to consider the interference effects caused by the correlation in the spatial disposition of individual scatterers.

The FM, SFM and PM were first studied in the forward problem framework in order to compare the theoretical and experimental BSCs. The relationship between BSC magnitude 
and scatterer concentration was addressed. The $B S C_{\text {exp }}$ magnitude was found to increase with the concentration at low scatterer concentrations, and then decreased at concentrations greater than 10\%. This pattern of BSC behavior versus the concentration was satisfactorily predicted by the SFM and the PM, but not by the FM. The SFM was the model that better matched the experiments at all the scatterer concentrations studied, especially in the 6-15 $\mathrm{MHz}$ frequency bandwidth.

The four scattering models were then examined to estimate the scatterer size and concentration from the experimentally measured $B S C_{\text {exp }}$. The GM did not yield accurate structural parameters, since it gave relative errors of approximately $74 \%$ in the radius and $90 \%$ in the concentration. The FM provided significant correlations between the estimated and true concentrations with $r^{2}$ around 0.96 at actual concentrations ranging from 1 and $12.5 \%$ (apart from the actual concentration of $5 \%$ ). However, the FM did not predict the concentration accurately at $\phi>12.5 \%$. The minimization routines used with the SFM and the PM were more complex than with the GM and FM, since the cost functions associated with these models often exhibited two local minima, corresponding to low and high scatterer concentration estimates. The global minimum did not always correspond to the actual parameters but one of the two local minima corresponded to the actual parameter. In order to solve this problem, it is possible to either (1) assume the medium to be dense a priori, or (2) associate the structural estimates with another quantitative parameter, such as the attenuation. With additional information of this kind, the SFM gave satisfactory concentration estimates with relative errors of less than $38 \%$ at actual concentrations ranging from 10 to $25 \%$. Future works should focus on the combination of the SFM with other quantitative parameters (attenuation, signal envelope statistics ${ }^{32}$ ) and on the use of higher frequencies. 


\section{APPENDIX A: ANALYTICAL EXPRESSION FOR THE STRUCTURE FACTOR}

The analytical expression for the structure factor can be obtained from the article of Wertheim $^{21}$ giving the analytical expression of the direct correlation function $c(r)$. Indeed, Percus-Yevick equation ${ }^{33}$ relates the pair correlation function (i.e. the radial distribution function) to the direct correlation function. Wertheim developed the analytical solution of the Percus-Yevick equation for hard spheres leading to ${ }^{21}$

$$
\begin{aligned}
-c(r) & =c_{0}+c_{1} \frac{r}{d}+c_{3}\left(\frac{r}{d}\right)^{3} & & \text { for } r \leq d \\
& =0 & & \text { for } r>d
\end{aligned}
$$

where $d$ is the hard-sphere diameter. The coefficients $c_{0}, c_{1}$ and $c_{3}$ are given by ${ }^{21}$

$$
\begin{aligned}
& c_{0}=\frac{(1+2 \phi)^{2}}{(1-\phi)^{4}} \\
& c_{1}=-\frac{6 \phi(1+\phi / 2)^{2}}{(1-\phi)^{4}} \\
& c_{3}=\frac{\phi}{2} c_{0}=\frac{\phi(1+2 \phi)^{2}}{2(1-\phi)^{4}}
\end{aligned}
$$

Note that the expression of $c_{0}$ is related to the inverse of the three-dimensional expression of the packing factor $W_{P Y}$ given in Eq. (6).

The Fourier transform of the structure factor $S$ is linked to the Fourier transform of the direct correlation function $C$ as follows: ${ }^{34}$

$$
S(k)=\frac{1}{1-m C(k)}
$$

with

$$
C(k)=4 \pi d^{3} \int_{0}^{1} r^{2} \frac{\sin (2 k r)}{2 k r} c(r) d r
$$

The structure factor $S$ was also calculated by computing the Fourier transform of a three-dimensional random distribution of particles as performed in $\operatorname{Ref}^{35}$ and was compared with the analytical expression of the structure factor (see Fig. 14a). The two curves show good agreement at large $k a>1$. In order to improve the analytical expression for small $k a$, 
we slightly modified the coefficient $c_{0}$ empirically as follows:

$$
c_{0}^{\prime}=\frac{(1+3 \phi)^{2}}{(1-\phi)^{4}}
$$

The inverse of $c_{0}^{\prime}$ corresponds to the modified packing factor expression proposed by Twersky ${ }^{36}$ with the variance parameter equals to 0 and with the shape parameter equals to 4. Since the new coefficients $c_{0}^{\prime}, c_{1}^{\prime}$ and $c_{3}^{\prime}$ verify the following equations: $c_{3}^{\prime}=(\phi / 2) c_{0}^{\prime}$ and $-c(d)=c_{0}+c_{1}+c_{3}=c_{0}^{\prime}+c_{1}^{\prime}+c_{3}^{\prime}$, we obtained the following expressions

$$
\begin{aligned}
& c_{1}^{\prime}=-\frac{8 \phi(1+\phi / 2)(1+\phi)}{(1-\phi)^{4}}, \\
& c_{3}^{\prime}=\frac{\phi}{2} c_{0}^{\prime}=\frac{\phi(1+3 \phi)^{2}}{2(1-\phi)^{4}} .
\end{aligned}
$$

Figure 14b) represents the comparison of the structure factors computed by the Fourier transform of a three-dimensional random distribution of particles and computed by the modified analytical methods based on the article of Wertheim ${ }^{21}$ using the coefficients given in Eq. (A5) and (A6). The two curves agrees well for high scatterer concentration even for small $k a$.

\section{ACKNOWLEDGMENTS}

This work was supported by the CNRS and by the French Agence Nationale de la Recherche (ANR), under grant ANR Tecsan 11-008-01. The author would like to thank the anonymous reviewers for their valuable suggestions, and Alain Busso, Eric Debieu and Stephan Devic at the Laboratory of Mechanics and Acoustics (LMA - CNRS) for their technical assistance.

\section{REFERENCES}

1 F. L. Lizzi, M. Greenebaum, E. J. Feleppa, and M. Elbaum, "Theoretical framework for spectrum analysis in ultrasonic tissue characterization", J. Acoust. Soc. Am. 73, 1366$1373(1983)$. 
${ }^{2}$ F. L. Lizzi, M. Ostromogilsky, E. J. Feleppa, M. C. Rorke, and M. M. Yaremko, "Relationship of ultrasonic spectral parameters to features of tissue microstructure", IEEE Trans. Ultrason. Ferroelect. Freq. Contr. 33 319-329 (1986).

3 E. J. Feleppa, F. L. Lizzi, D. J. Coleman, and M. M. Yaremko, "Diagnostic spectrum analysis in ophthalmology: a physical perspective", Ultrasound Med. Biol. 12 623-631 (1986).

4 E. J. Feleppa, T. Liu, A. Kalisz, M. C. Shao, N. Fleshner, and V. Reuter, "Ultrasonic spectral-parameter imaging of the prostate", Int. J. Imag. Syst. Technol. 8, 11-25 (1997).

5 M. L. Oelze, J. F. Zachary and W. D. O’Brien, "Characterization of tissue microstructure using ultrasonic backscatter: Theory and technique for optimization using a Gaussian form factor", J. Acoust. Soc. Am. 112 1202-1211 (2002)

6 M. L. Oelze, W. D. O’Brien, J. P. Blue, and J. F. Zachary, "Differentiation and characterization of rat mammary fibroadenomas and $4 \mathrm{~T} 1$ mouse carcinomas using quantitative ultrasound imaging", IEEE Trans. Med. Imaging 23 764-771 (2004).

7 M. L. Oelze and W. D. O'Brien, "Application of three scattering models to characterization of solid tumors in mice", Ultrasonic Imaging 28 83-96 (2006).

8 M. C. Kolios, G. J. Czarnota, M. Lee, J. W. Hunt and M. D. Sherar, "Ultrasonic spectral parameter characterization of apoptosis", Ultrasound Med. Biol. 28 589-597 (2002).

9 J. Mamou, A. Coron, M. Hata, J. Machi, E. Yanagihara, P. Laugier and E. Feleppa, "Three-dimensional high-frequency characterization of cancerous lymph nodes", Ultrasound Med. Biol. 36 361-375 (2010).

10 M. F. Insana, R. F. Wagner, D. G. Brown and T. J. Hall, "Describing small-scale structure in random media using pulse-echo ultrasound", J. Acoust. Soc. Am. 87 179-192 (1990).

11 R. M. Vlad, R. K. Saha, N. M. Alajez, S. Ranieari, G. J. Czarnota and M. C. Kolios, "An increase in cellular size variance contributes to the increase in ultrasound backscatter during cell death", Ultrasound in Medicine \& Biology 9 1546-1558 (2010).

12 L. Y. L. Mo and R. S. C. Cobbold RSC, "Theoretical models of ultrasonic scattering in blood", in Ultrasonic Scattering in Biological Tissues, edited by K. K. Shung and G. A. 
Thieme (CRC, Boca Raton, FL, 1993), Chap.5, pp. 125-170.

13 V. Twersky, "Low-frequency scattering by correlated distributions of randomly oriented particles", J. Acoust. Soc. Am. 81 1609-1618 (1987).

14 K. K. Shung, "On the ultrasound scattering from blood as a function of hematocrit", IEEE Trans. Ultras., Ferroelect., Freq. Contr. SU-26, 327-331 (1982).

15 D. Savery and G. Cloutier, "A point process approach to assess the frequency dependence of ultrasound backscattering by aggregating red blood cells", J. Acoust. Soc. Am., vol. 110, no. 6, pp. 32523262, 2001.

16 I. Fontaine, D. Savery and G. Cloutier, "Simulation of ultrasound backscattering by red blood cell aggregates: effect of shear rate and anisotropy", Biophysical Journal, vol.82, pp. 1696-1710, 2002.

17 M. F. Insana and D. G. Brown, "Acoustic scattering theory applied to soft biological tissues", in Ultrasonic Scattering in Biological Tissues, edited by K. K. Shung and G. A. Thieme (CRC, Boca Raton, FL, 1993), Chap.4, pp. 76-124.

18 J. J. Faran, "Sound Scattering by Solid Cylinders and Spheres", J. Acoust. Soc. Amer. 121 405-418 (1951).

19 Franceschini E., Metzger B. and Cloutier G., "Forward problem study of an effective medium model for ultrasound blood characterization", IEEE Trans. on Ultrason., Ferroelect., Freq. Contr. 58 2668-2679 (2011).

20 I. Fontaine, M. Bertrand and G. Cloutier G, "A system-based approach to modeling the ultrasound signal backscattered by red blood cells", Biophysical Journal 77 2387-2399 (1999).

21 M. S. Wertheim, "Exact solution of the Percus-Yevick integral equation for hard spheres", Physical Review Letters 10 321-323 (1963).

${ }^{22}$ R. E. Baddour and M. C. Kolios, "The fluid and elastic nature of nucleated cells: implications from the cellular backscatter response", J. Acoust. Soc. Am. 121 EL16-22 (2007).

23 T. E. Doyle, K. H. Warnick and B. L. Carruth, "Histology-based simulations for the 
ultrasonic detection of microscopic cancer in vivo", J. Acoust. Soc. Am. 122 EL210-216 (2007).

24 R. Kuc a,d M. Schwartz, "Estimating the acoustic attenuation coefficient slope for liver from reflected ultrasound signals", IEEE Trans Sonics Ultrasonics SU-26, 353-362 (1979). S. H. Wang and K. K. Shung, "An approach for measuring ultrasonic backscattering from biological tissues with focused transducers, IEEE Trans. Biomed. Eng. 44, 549554 (1997).

${ }^{26}$ E. Franceschini, F. T. H. Yu, F. Destrempes and G. Cloutier, "Ultrasound characterization of red blood cell aggregation with intervening attenuating tissue-mimicking phantoms". J. Acoust. Soc. Am. 127 1104-1115 (2010).

27 J. J. Anderson, M. T. Herd, M. R. King, A. Haak, Z. T. Hafez, J. Song, M. L. Oelze, E. L. Madsen, J. A. Zagzebski, W. D. O’Brien, T. J. Hall, "Interlaboratory comparison of backscatter coefficient estimates for tissue-mimicking phantoms", Ultrasonic imaging 32 48-64 (2010).

28 O. Falou, M. Rui, A. E. Kaffas, J. C. Kumaradas, and M. C. Kolios, "The measurement of ultrasound scattering from individual micron-sized objects and its application in single cell scattering," J. Acoust. Soc. Am. 128 894-902 (2010).

29 M. Teisseire, A. Han, R. Abuhabsah, J. P. Blue, Jr., S. Sarwate and W. D. O’Brien, "Ultrasonic backscatter coefficient quantitative estimates from chinese hamster ovary cell pellet biophantoms", J. Acoust. Soc. Am. 128 3175-3180 (2010).

30 M. R. King, J. J. Anderson, M.-T. Herd, D. Ma, A. Haak, L. A. Wirtzfeld, E. L. Madsen, J. A. Zagzebski, M. L. Oelze, T. J. Hall and W. D. O'Brien, "Ultrasonic backscatterer coefficients for weakly scattering, agar spheres in agar phantoms", J. Acoust. Soc. Am. 128 903-908 (2010).

31 J.-F. Chen and J. A. Zagzebski, "Frequency dependence of backscatterer coefficient versus scatterer volume fraction", IEEE Trans. Ultras., Ferroelect., Freq. Contr. 43 345-353 (1996).

32 R. K. Saha and M. C. Kolios, "Effects of cell spatial organization and size distribution on ultrasound backscattering", IEEE Trans. Ultras., Ferroelect., Freq. Contr. 58, 2118-2131 
(2011).

33 J. K. Percus and G. J. Yevick, "Analysis of classical statistical mechanics by means of collective coordinates", Physical Review 110, 1-13 (1958).

34 N. W. Ashcroft, "Structure and resistivity of liquid metals", Physical Review 145, 83-90 (1966).

35 R. K. Saha, E. Franceschini and G. Cloutier, "Assessment of accuracy of the structurefactor-size-estimator method in determining red blood cell aggregate size from ultrasound spectral backscatter coefficient", J. Acoust. Soc. Amer. 129, 2269-2277 (2011).

36 V. Twersky, "Low-frequency scattering by mixtures of correlated nonspherical particles", J. Acoust. Soc. Am. 84 409-415 (1988). 


\section{TABLE CAPTIONS}

Table I. Summary of the parameters used to calculate the theoretical BSC response of the polyamide microsphere. Comparisons with parameters for human acute myeloid leukemia cell OCI-AML-5 used in Baddour and Kolios. ${ }^{22}$ 
TABLE I.

\begin{tabular}{|c|c|c|c|}
\hline & $\begin{array}{c}\text { Polyamide } \\
\text { microsphere }\end{array}$ & $\begin{array}{c}\text { OCI-AML-5 } \\
\text { nucleus }\end{array}$ & $\begin{array}{c}\text { OCI-AML-5 } \\
\text { cell }\end{array}$ \\
\hline Radius $a(\mu \mathrm{m})$ & 6 & 4.55 & 5.75 \\
\hline Sound speed $c(\mathrm{~m} / \mathrm{s})$ & 2300 & 1503 & 1535 \\
\hline Density $\rho\left(\mathrm{kg} / \mathrm{m}^{3}\right)$ & 1030 & 1430 & 1240 \\
\hline Impedance $z$ (MRayl) & 2.37 & 2.15 & 1.90 \\
\hline Poisson's ratio & 0.42 & 0.42 & 0.487 \\
\hline
\end{tabular}


Figure 1. (Color online) (a) Typical aspect of the logarithm of the cost function $F_{F M}(a, \phi)$ for the FM obtained from the experiments with actual microsphere radius of $6 \mu \mathrm{m}$ and actual concentration of $2.5 \%$. The logarithm is shown here in order to enhance the visual contrast. Black rectangle indicates the ROI used to compute the gradient in subplot (c). (b) Typical aspects of the function $\log \left(F_{F M}(a, \phi)\right)$ obtained with several fixed values of $a$. These cost functions have a single minimum. (c) Direction and relative magnitude of the gradient in the ROI around the values of the actual parameters.

Figure 2. (Color online) (a) Typical aspect of the logarithm of the cost function $F_{S F M}(a, \phi)$ for the SFM obtained from the experiments with actual microsphere radius of $6 \mu \mathrm{m}$ and actual concentration of $20 \%$. The logarithm is shown here in order to enhance the visual contrast. This cost function has two possible solutions $\left(a_{1}^{*}, \phi_{1}^{*}\right)$ and $\left(a_{2}^{*}, \phi_{2}^{*}\right)$. Black rectangle indicates the ROI used to compute the gradient in subplot (c). (b) Typical aspects of the function $\log \left(F_{S F M}(a, \phi)\right)$ obtained with several fixed values of $a$. These cost functions can display either one or two minima. (c) Direction and relative magnitude of the gradient in the ROI around the values of the actual parameters.

Figure 3. Normalized histogram of the microsphere radii.

Figure 4. (Color online) Diagram of the experimental set-up used to perform the attenuation measurement.

Figure 5. Attenuation $\alpha_{p h}$ of the tissue-mimicking phantoms as a function of the actual concentration.

Figure 6. (Color online) BSCs at scatterer concentrations of 1, 5 and $10 \%$. (a) Comparison between the measured BSCs and the FM predictions. (b) Comparison between the measured BSCs and the SFM and PM predictions. 
Figure 7. (Color online) BSCs at scatterer concentrations of 15 and $25 \%$. (a) Comparison between the measured BSCs and the FM predictions. (b) Comparison between the measured BSCs and the SFM and PM predictions.

Figure 8. (Color online) Comparison between the measured and theoretical mean BSC versus the scatterer concentration (a) in the 6 to $15 \mathrm{MHz}$ frequency range and (b) in the 15 to $22 \mathrm{MHz}$ frequency range.

Figure 9. (Color online) Spectral slopes obtained in the 6-22 MHs frequency bandwidth as a function of the actual concentration in the experiments and with the three theoretical scattering models (FM, SFM and PM).

Figure 10. (Color online) a) Values of $a^{*}$ and $\phi^{*}$ estimated by the GM and the FM versus the actual concentration. Also represented are actual values of $a$ and $\phi$. (b) Corresponding relative errors of $a^{*}$ and $\phi^{*}$.

Figure 11. (Color online) a) Parameter estimates using the SFM versus the actual concentration. The triangular and the diamond-shaped symbols represent the possible solutions $\left(a_{1}^{*}, \phi_{1}^{*}\right)$ and $\left(a_{2}^{*}, \phi_{2}^{*}\right)$, respectively, with an initial value of the concentration $\phi^{\text {init }}=0.001$ and $\phi^{\text {init }}=0.74$, respectively, at the beginning of the minimization routine. The circular symbols give the global minimum, i.e. the solution $\left(a^{*}, \phi^{*}\right)$. Also represented in dashed lines are actual values of $a$ and $\phi$. (b) Corresponding relative errors.

Figure 12. (Color online) a) Parameter estimates using the PM versus the actual concentration. The triangular and the diamond-shaped symbols represent the possible solutions $\left(a_{1}^{*}, \phi_{1}^{*}\right)$ and $\left(a_{2}^{*}, \phi_{2}^{*}\right)$, respectively, with an initial value of the concentration $\phi^{i n i t}=0.001$ and $\phi^{i n i t}=0.74$, respectively, at the beginning of the minimization routine. The circular symbols give the global minimum, i.e. the solution $\left(a^{*}, \phi^{*}\right)$. Also represented in dashed lines are actual values of $a$ and $\phi$. (b) Corresponding relative errors. 
Figure 13. (Color online) a) $B S C_{S F M}$ magnitude versus the concentration computed with the SFM for several fixed microsphere radii ranging from 5 to $7 \mu \mathrm{m}$. b) Experimental $B S C_{\text {exp }}$ at a scatterer concentration of $20 \%$ and the theoretical $B S C_{S F M}$ computed with two possible solutions $\left(a_{1}^{*}=6.5 \mu \mathrm{m}, \phi_{1}^{*}=5.69 \%\right)$ and $\left(a_{2}^{*}=6.9 \mu \mathrm{m}\right.$, $\phi_{2}^{*}=22.50 \%$ ). c) Theoretical $B S C_{S F M}$ computed with the SFM for two scatterer concentrations of $5 \%$ and $20 \%$ with microsphere radii of $5.5 \mu \mathrm{m}$ and $6 \mu \mathrm{m}$.

Figure 14. Comparison between structure factors at three different concentrations 5, 15 and $25 \%$, computed by the Fourier transform (FT) of a three-dimensional random distribution of particles ${ }^{35}$ and computed by two analytical methods based on the article of Wertheim ${ }^{21}$ (a) using the coefficients given in Eq. (A2) and (b) using the coefficients given in Eq. (A5) and (A6). 
(a)

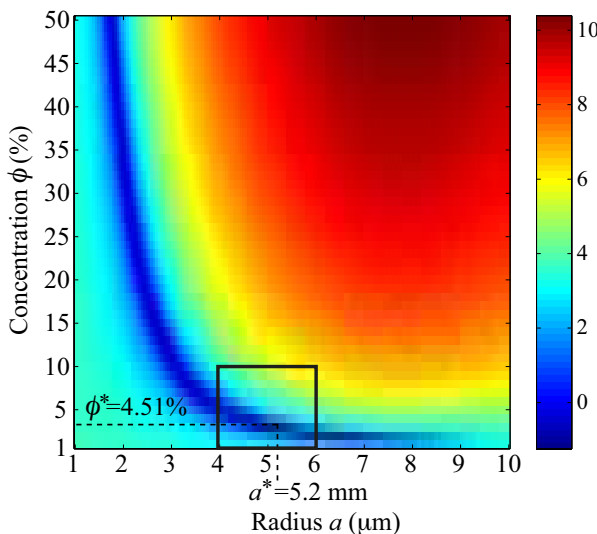

(b)

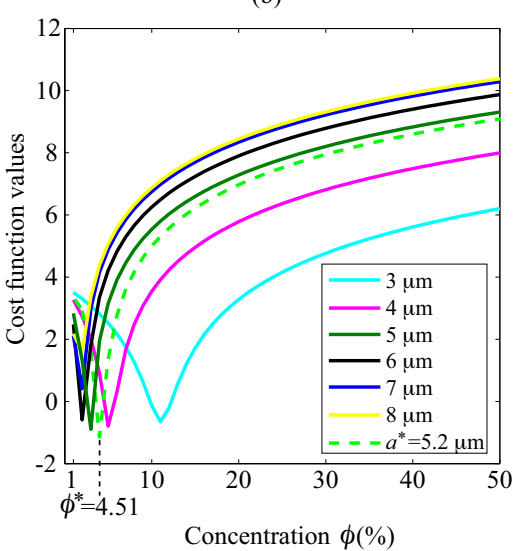

(c)

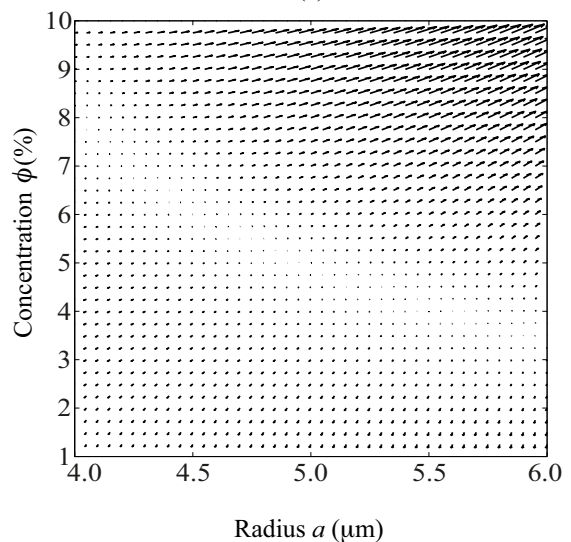

FIG. 1. 
(a) $a_{2}^{*}=6.9 \mu \mathrm{m}$

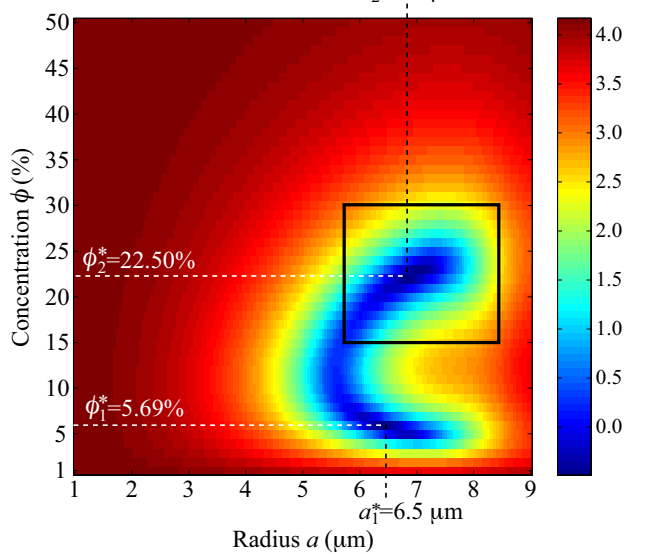

(b)
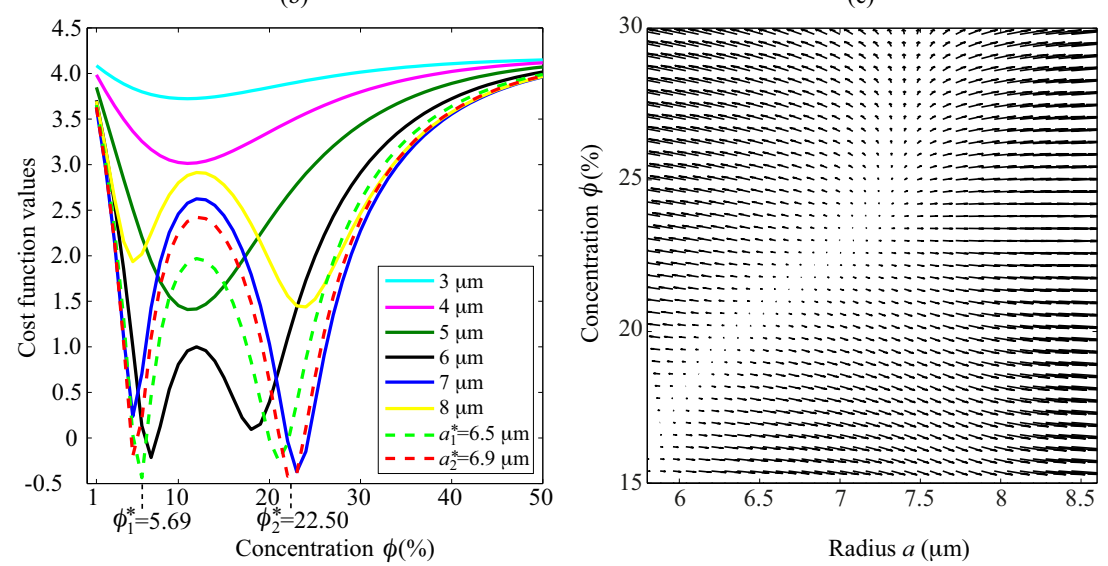

FIG. 2. 


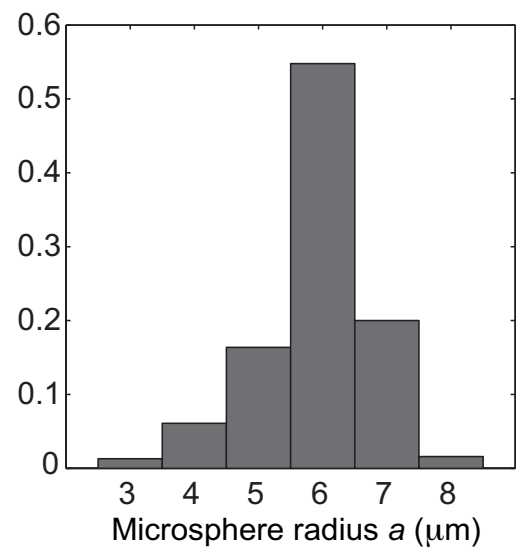

FIG. 3. 


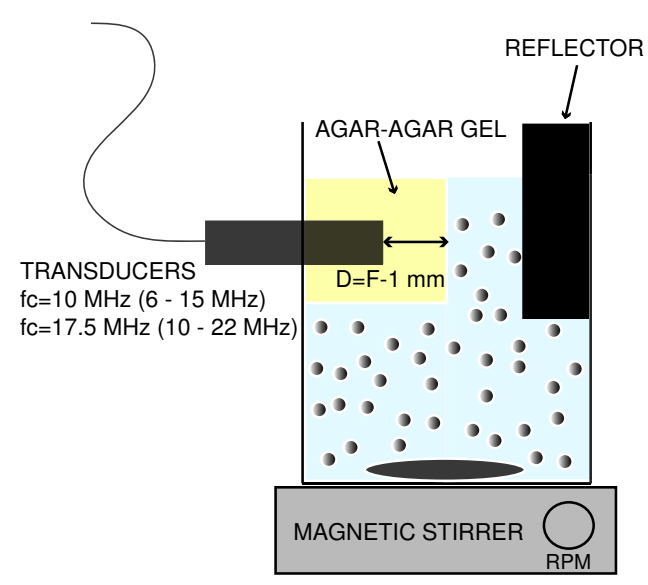

FIG. 4. 


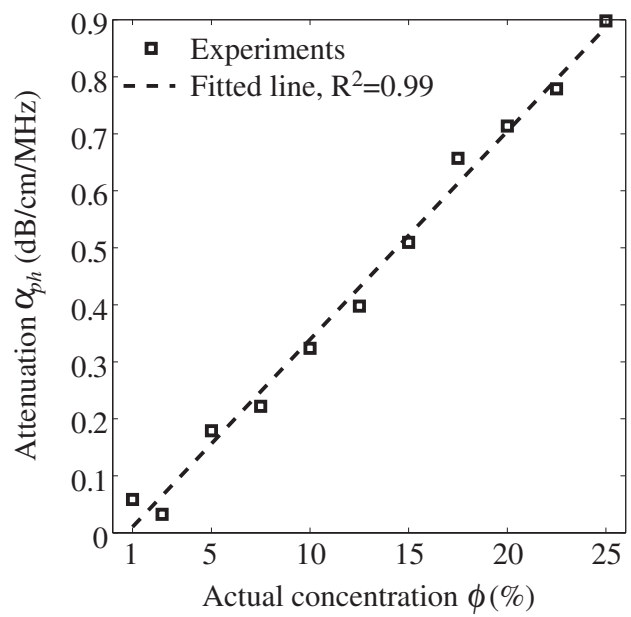

FIG. 5. 

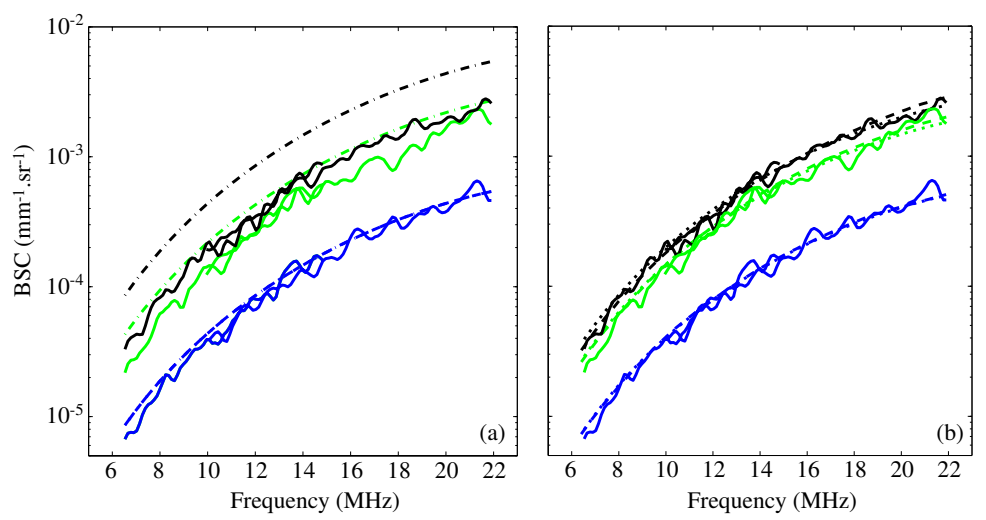

- $10 \mathrm{MHz}, \quad \phi=1 \%$ $17.5 \mathrm{MHz}, \phi=1 \%$

- FM, $\phi=1 \%$

.. PM, $\phi=1 \%$

- SFM, $\phi=1 \%$

$10 \mathrm{MHz}, \quad \phi=5 \%$

$17.5 \mathrm{MHz}, \phi=5 \%$

FM, $\phi=5 \%$

PM, $\phi=5 \%$

- SFM, $\phi=5 \%$

- $10 \mathrm{MHz}, \quad \phi=10 \%$

- $17.5 \mathrm{MHz}, \phi=10 \%$

- FM, $\phi=10 \%$

... PM, $\phi=10 \%$

- - SFM, $\phi=10 \%$

FIG. 6. 

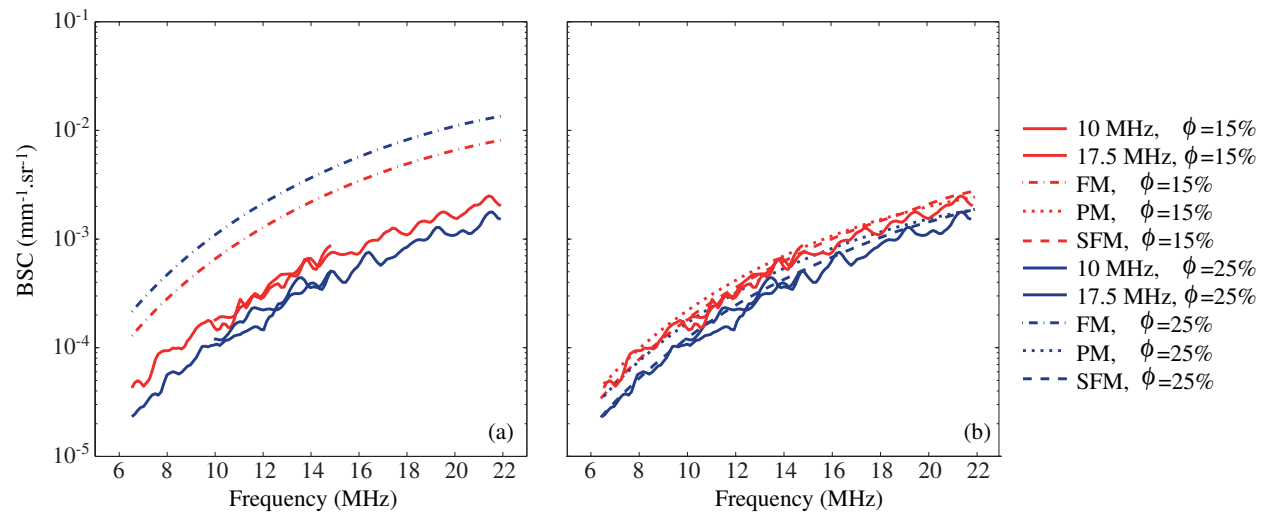

FIG. 7. 

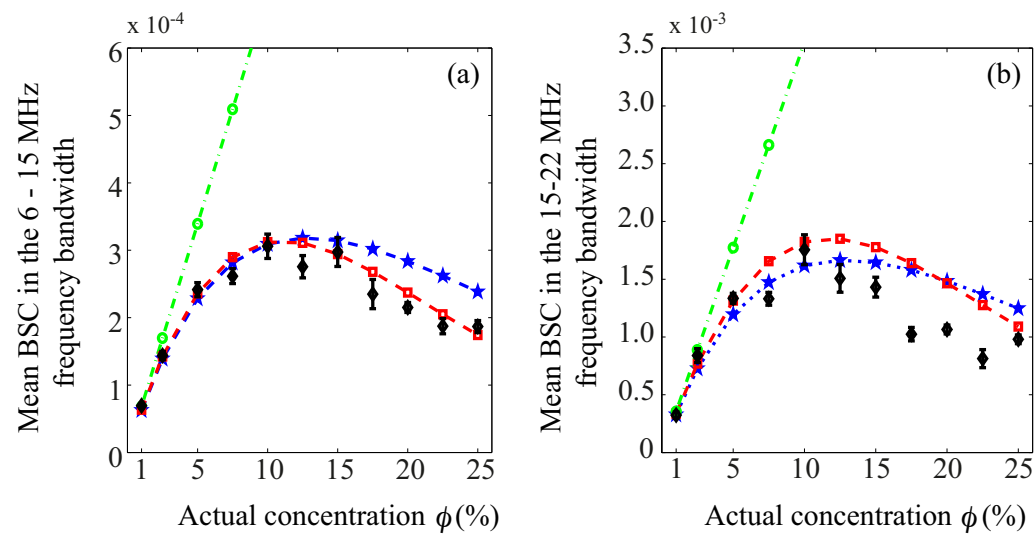

$\checkmark$ Experiments

- - Faran Model (FM)

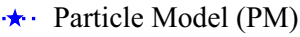

- в - Structure Factor Model (SFM)

FIG. 8. 


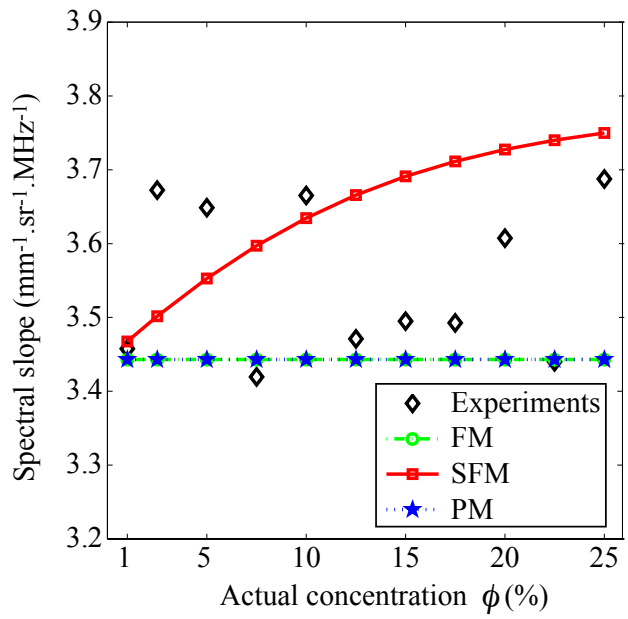

FIG. 9. 

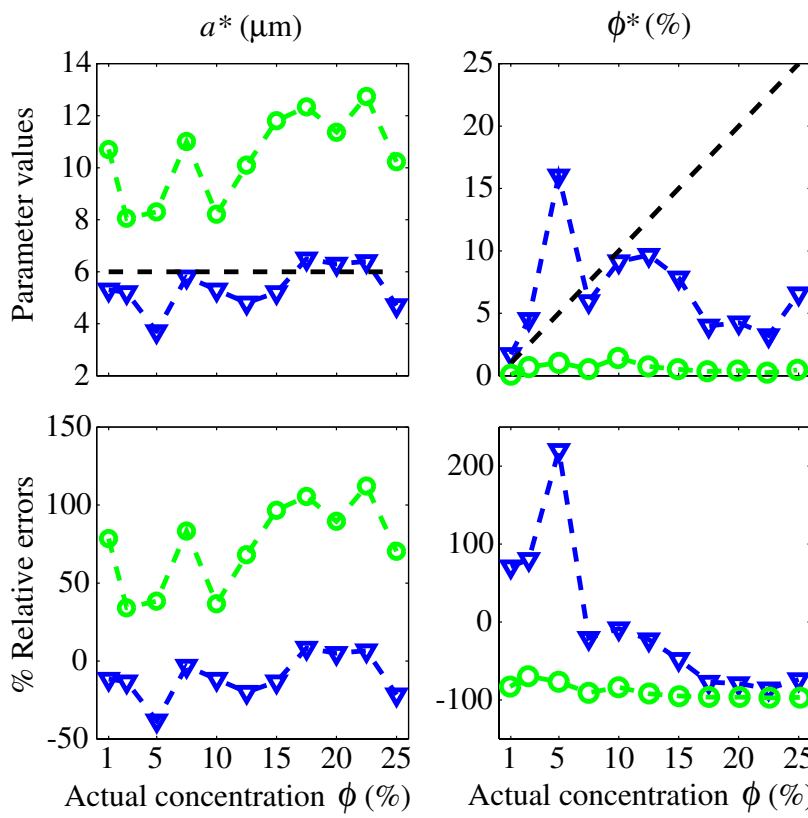

- - - Actual parameters

- $\boldsymbol{\nabla}$ - Estimated parameters

with the Faran Model

- $\odot$ - Estimated parameters

with the Gaussian Model

FIG. 10. 

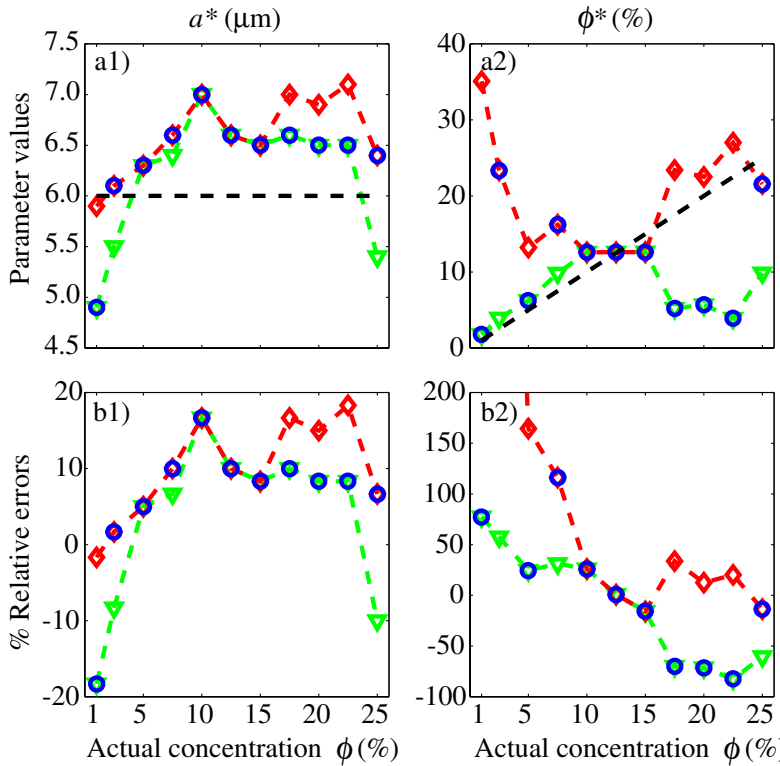

- - - Actual parameters Local minimum with a low initial concentration $\phi^{\text {init }}=0.001$

$-\diamond-$ Local minimum with a high initial concentration $\phi^{\text {init }}=0.74$

- Global minimum

FIG. 11. 

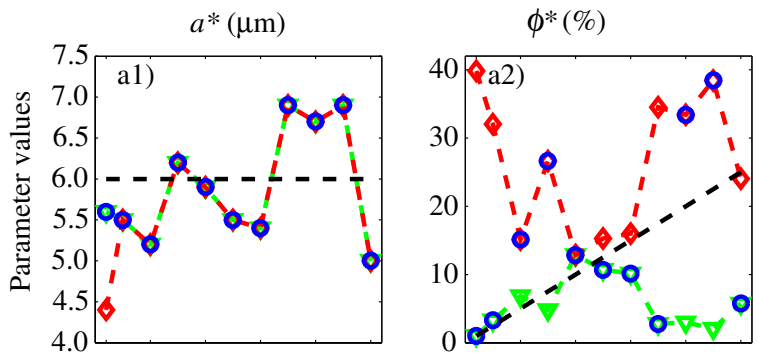

- - - Actual parameters

$-\nabla-$ Local minimum with a low initial concentration $\phi^{\text {init }}=0.001$
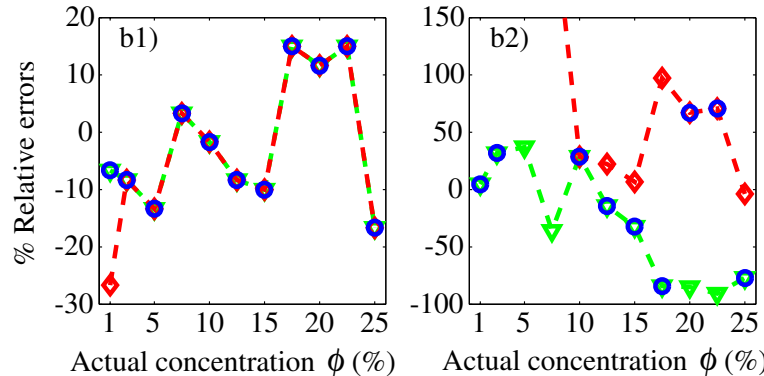

- $\diamond$ - Local minimum with a high

initial concentration $\phi^{\text {init }}=0.74$

- Global minimum

FIG. 12. 

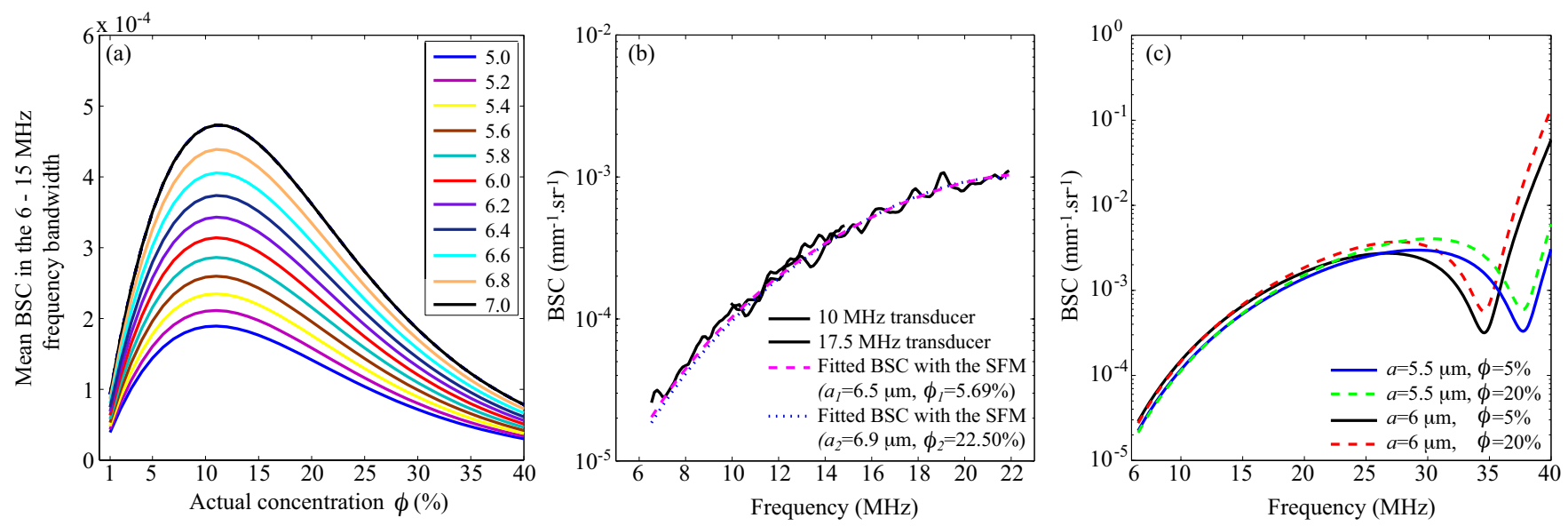

FIG. 13. 

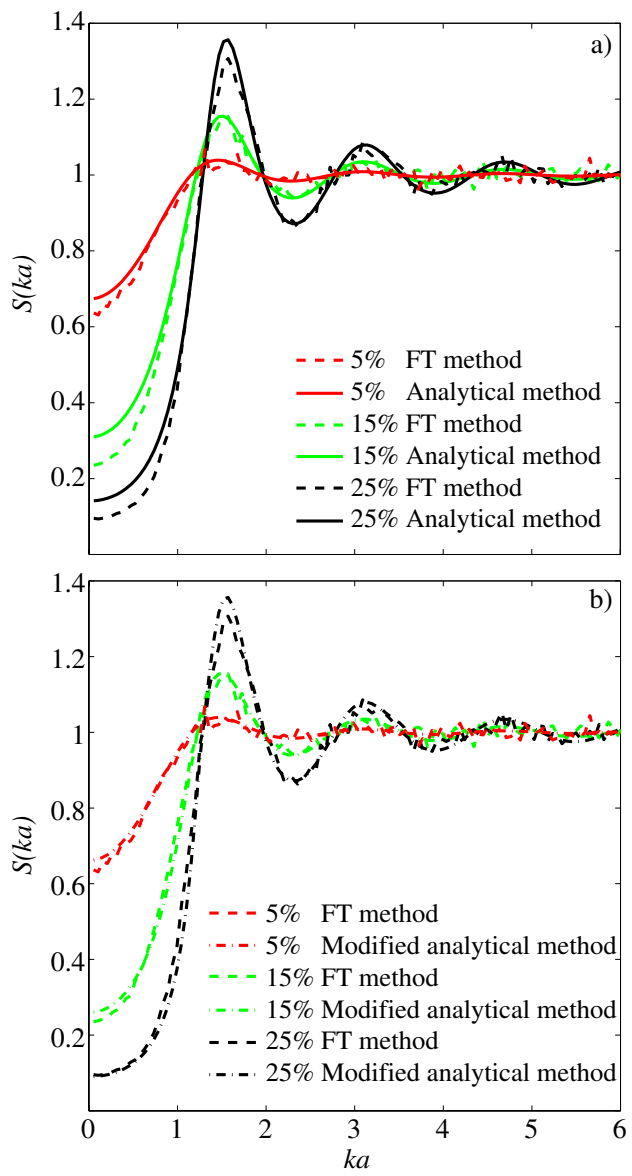

FIG. 14. 